\title{
Risikofaktoren für die venöse Thromboembolie
}

\section{Studien in den letzten Jahren haben eindeutig gezeigt, dass es Gemein- samkeiten in der Pathogenese von venösen und arteriellen Thrombo- embolien gibt. Gibt es auch gemein- same Risikofaktoren?}

— In der Copenhagen-City-Heart-Studie, einer prospektiven Kohortenstudie, die von 1976 bis 2007 lief, wurden die Risikofaktoren für venöse Thromboembolien untersucht. Die Daten wurden dem nationalen Register entnommen. 969 von 18954 Probanden (mittlere Beobachtungsdauer: 19,5 Jahre) erlitten eine tiefe Beinvenenthrombose. Dies entspricht einer Inzidenzrate von 2,69. Folgende Risikofaktoren waren mit dem Auftreten von venösen Thromboembolien assoziiert:

- Body-Mass-Index $>35 \mathrm{~kg} / \mathrm{m}^{2}$ : Hazard Ratio $(\mathrm{HR})=2,10$ (vs. BMI $<20$ - Rauchen > 25 g Tabak pro Tag: HR = 1,52 (vs. Nichtraucher),

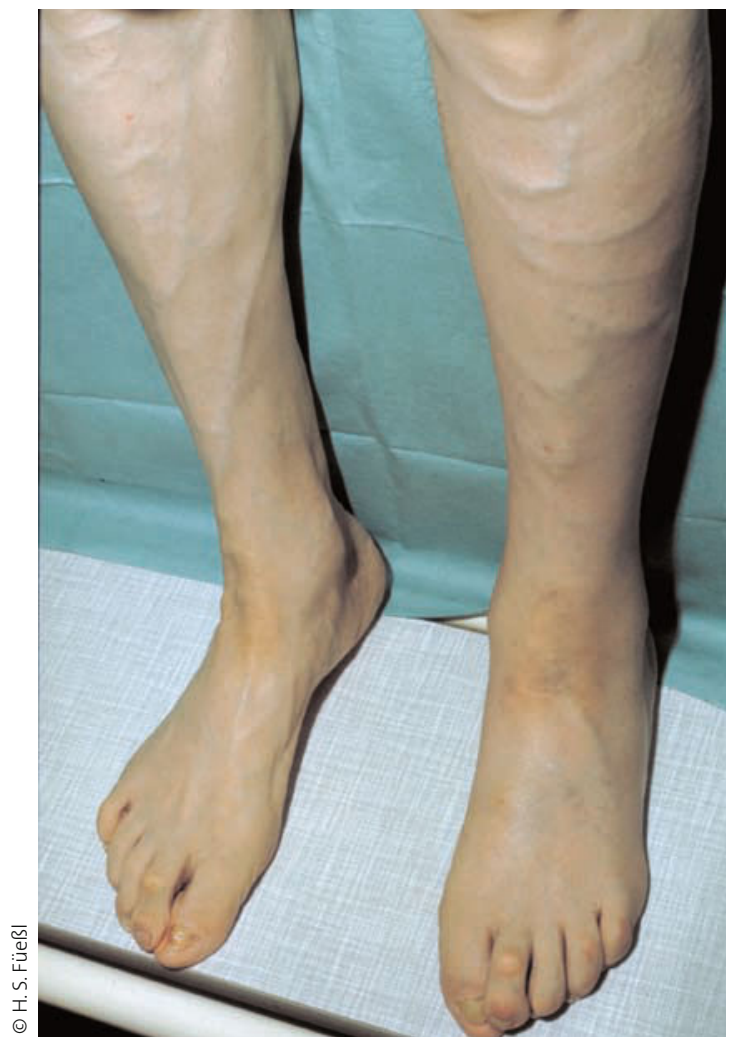

4 Tiefe Beinvenenthrombose: bei Rauchern und Adipösen häufiger.
- männliches Geschlecht: HR = 1,24 (vs. Frauen),

- mittleres Einkommen: HR = 0,82 (vs. geringes Einkommen) und

- diastolischer Blutdruck > $100 \mathrm{mmHg}$ : $\mathrm{HR}=1,34$ (vs. $<80 \mathrm{mmHg}$ ).

Damit waren Übergewicht und Rauchen die beiden bedeutendsten Risikofaktoren für die Entstehung einer venösen Thromboembolie. Andere Risikofaktoren wie Gesamtcholesterin-, HDL- und LDL-Cholesterin- und Triglyzeridspiegel sowie Diabetes mellitus waren dagegen nicht mit dem Auftreten einer venösen Thromboembolie assoziiert.

\section{Kommentar}

Dies ist die größte prospektive Studie zu Risikofaktoren für eine venöse Thromboembolie. Es zeigte sich, dass die venöse Thromboembolie und die Atherothrombose gemeinsame Risikofaktoren haben. Überraschend war, dass es keine Assoziation zwischen einer Fettstoffwechselstörung und dem Auftreten von Thromboembolien $g a b$

- A. Holst A et al.

Risk Factors for Venous Thromboembolism - Results From the Copenhagen City Heart Study. Circulation 121 (2010) 1896-1903.

\section{Sie lesen Qualität}

Damit das auch so bleibt, befragen wir Sie in Kooperation mit tns infratest in den nächsten Wochen.

Ihr Urteil ist uns wichtig. Bitte nehmen Sie teil! 